\title{
Hierarchical patterning by multi-step micro-imprinting with layered materials
}

\author{
Fujio Tsumori ${ }^{1, *}$, Lijan Shen ${ }^{2}$, Toshiko Osada ${ }^{1}$, and Hideshi Miura ${ }^{1}$ \\ 1 Dept. of Mech. Eng., Kyushu Univ., 744 Motooka, Nishi-ku, Fukuoka 819-0395, Japan \\ 2 Dept. of Mech. Eng., Grad. School of Eng., Kyushu Univ., 744 Motooka, Nishi-ku, Fukuoka 819-0395, Japan
}

Received 26 January 2015 / Accepted 24 February 2015

\begin{abstract}
The objective of the work reported in this paper is to create multi-scale and hierarchical surface structures using a simple imprinting process. The hierarchical structures can be fabricated with only simple patterned molds by proposed multi-step imprinting process, instead of using a high cost hierarchical-patterned mold. In the proposed process, the starting material is a layered sheet material. The layered sheet is pressed by a mold with a finer pattern, and subsequently pressed by a mold with a rougher pattern. A pure polymer sheet is employed as the upper layer, which will be removed during heating processes as a sacrificed layer, while the lower layer is a compound material of polymer and ceramic powder. After heating process, ceramic compact is sintered and formed a full-dense patterned sheet. By the proposed multi-step imprinting process with a layered material, ceramic sheets with micro hierarchical pattern can be fabricated with low cost. In the present work, poly(vinyl alcohol) (PVA) was prepared as the upper layer, and a compound material of alumina powder and PVA as a lower layer. Molds with finer and rougher patterns were also prepared for multi-step imprinting. As a result, a hierarchical structure on a thin ceramic sheet could be fabricated.
\end{abstract}

Key words: Imprint, Ceramics, Hydrophobic surface, Multi-scale patterning, Bio mimicry

\section{Introduction}

Hierarchical surface patterns are found in the natural world, and they show lots of different functions; e.g., surface structures on moth eye eliminate reflection [1]; and lotus leaves have micro-scale hierarchical pattern, which causes super hydrophobicity [2]. In this paper, we targeted such kind of bio-mimic surface structure, and newly developed a simple imprinting process to obtain the bio-mimic functional surface.

Our process is based on nano imprint lithography, which has been proposed by Stephen Chou in 1995; a sub-100 nm patterns were demonstrated by physically deforming a thermoplastic material as a substrate in a temperature and pressure controlled printing process with a fine mold [3]. Nano imprint lithography is considered as one of the efficient methods to fabricate nano-scale patterns on surface of polymer material. It is also a high-throughput and low-cost method.

In recent years, some improvement of the imprinting process has been reported. Authors proposed a combined process with powder metallurgy as micro powder imprint method [4-9]. In this process, a compound material, mixture of ceramic powder and a polymer binder system, is used for a working material. The compound sheet is pressed by a mold with micrometer scale patterns, and subsequently the polymer

*e-mail: tsumori@mech.kyushu-u.ac.jp binder materials are totally removed during debinding and sintering processes at elevated temperatures. The micro powder imprint process has been applied to fabricate micro-scale patterns on surface of an yttria-stabilized zirconia ceramic sheet, which may be available for an electrolyte sheet of solid oxide fuel cell [10]. To improve the cell efficiency, micro-scale patterns on both sides of sheet are effective, so that bilayer imprinting has been proposed [10]. Bilayer imprinting is a method of pressing a two-layered sheets of different materials at one time, therefore the pattern is left not only on the surface of upper sheet but also on the interface of the upper and lower layers. The viscoelastic character of these two kinds of layers and the thickness of the upper layer are essential parameters for the bilayer imprinting [11].

The multi-step micro imprinting process has been also proposed recently [10]. In the work reported in this paper, the bilayer imprinting method was adopted for the bio-mimic hierarchical surface structure. As the starting sheet material, a stacked sheet of polymer sheet and a ceramic compound sheet was prepared, and two step imprinting with fine and rough molds was carried out. As a result, the micro patterns on the interface between two layers were obtained. After heating process for removing the polymer material and for sintering into dense ceramic structure, we can obtain a ceramic sheet with hierarchical surface pattern. 


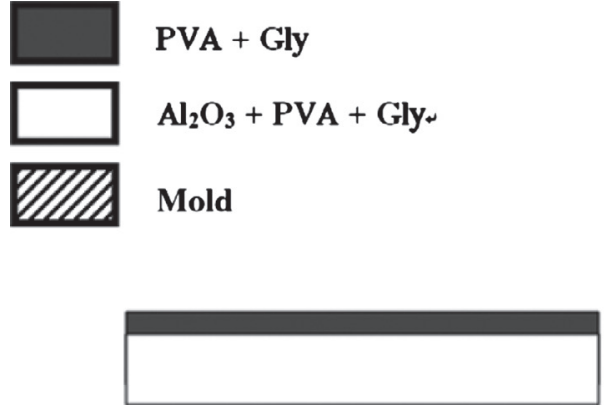

(a)

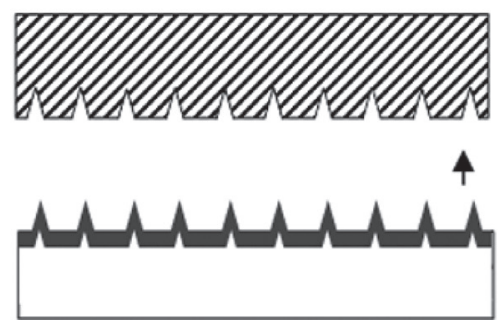

(b)

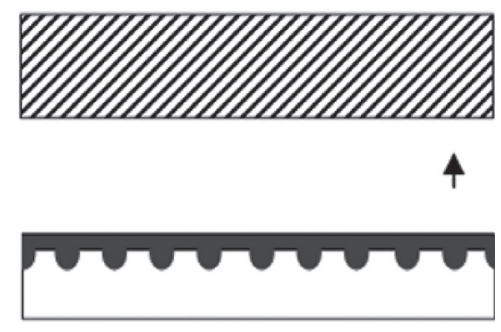

(c)

Figure 1. Schematic flow of the present process: (a) bilayer-substrate preparing, (b) the first micro-imprinting with fine patterns, and (c) the second pressing with flat surface.

\section{Experiment procedure}

\subsection{Preparation of slurry}

At first, sheet materials were prepared to conduct the bilayer imprinting. The starting materials were as follows: $\mathrm{Al}_{2} \mathrm{O}_{3}$ powder (mean diameter: $0.1 \mu \mathrm{m}$, Taimei Chemical Co.), poly-vinyl alcohol (degree of polymerization: 500, degree of saponification: $86-90 \%$ ) as a polymer binder material, and glycerin as plasticizer. Mixing ratio of $\mathrm{Al}_{2} \mathrm{O}_{3}$ compound sheet was $40 \mathrm{vol} \%$ of powder, $30 \mathrm{vol} \%$ of PVA, and $30 \mathrm{vol} \%$ of glycerin. First, the $\mathrm{Al}_{2} \mathrm{O}_{3}$ powder was put into pure water. Then, ultrasonic of $45 \mathrm{kHz}$ was applied for $10 \mathrm{~min}$ to get good dispersion. After PVA solution and glycerin were added, the mixture was stirred by $10 \mathrm{~min}$ by a magnetic stirrer.

A polymer sheet without $\mathrm{Al}_{2} \mathrm{O}_{3}$ powder was also prepared. The composition of the polymer sheet was that $90 \mathrm{vol} \%$ of PVA and $10 \mathrm{vol} \%$ of glycerin. The compound sheet was used as a lower layer, and the sheet without powder as upper one. Both sheets were stacked as a layered sheet for the layered imprinting process.

\subsection{Sheet casting}

A sheet casting process was employed to obtain sheets material. The prepared slurry in the above section was applied on polyethylene terephthalate sheet thinly and uniformly by a table coater (Mitsui Seiki Electric Co.). And then, the applied slurry was kept at $80{ }^{\circ} \mathrm{C}$ for $60 \mathrm{~min}$ in an oven to dry out water. After drying, $\mathrm{Al}_{2} \mathrm{O}_{3}$ compound sheet was obtained. Thickness of the obtained sheets was controlled to be about $80 \mu \mathrm{m}$.

We also prepared pure polymer sheets without powder as the upper layer by the same method. The thickness of the polymer sheet was controlled to $2.8,2.3,1.8$, and $1.5 \mu \mathrm{m}$.

\subsection{Bilayer imprinting}

Figure 1 shows schematic flow of the present bilayer imprinting process. Two sheets, a polymer sheet and an $\mathrm{Al}_{2} \mathrm{O}_{3}$ compound sheet, were stacked as a layered-material as shown in Figure 1a. Next, imprinting was performed with a

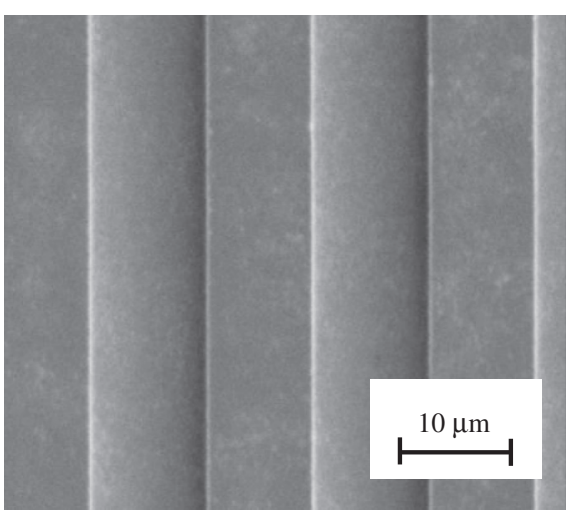

Figure 2. SEM image of silicon mold: line \& space patterns with $20 \mu \mathrm{m}$ pitch and $5 \mu \mathrm{m}$ depth.

mold (Figure 1b). The mold pattern was transcribed on the upper layer's surface, and also interface pattern appeared between upper and lower layers. Finally, the imprinted pattern was pressed with a flat surface as shown in Figure 1c. The author has already reported how this interfacial pattern was formed by the imprint process to layered material [11]. The pattern is influenced by formability of both upper and lower layers. In this figure, we show the pressing process with a flat surface. If a mold with a rougher pattern were used instead of the flat surface, we could obtain a multi-scaled surface pattern.

The imprinting equipment (MINI-1000HC, MARUNI) was used for the imprinting process. There were two kinds of important parameters, temperature and pressure. In the present experiment, the imprinting temperature was set to be $102{ }^{\circ} \mathrm{C}$, which was determined by preliminary experiment. The pressure for the first imprinting with fine mold was also set to $48 \mathrm{MPa}$, and for the second one with rougher mold was set to $60 \mathrm{MPa}$. Figure 2 shows SEM image of the silicon mold for the first imprinting process, which had line \& space patterns with $20 \mu \mathrm{m}$ in pitch and $5 \mu \mathrm{m}$ in depth. Figure 3 shows the rougher mold for the second imprinting. The rougher mold was made of polyimide by laser machining, which had $100 \mu \mathrm{m}$ pitch and $80 \mu \mathrm{m}$ depth. 

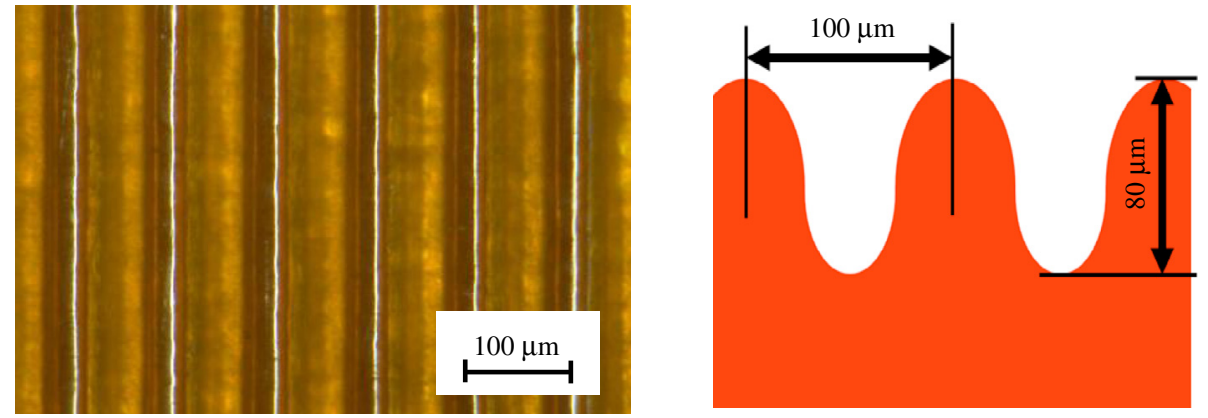

Figure 3. Optical micro photo and schematic image of the mold for the second imprinting: $100 \mu \mathrm{m}$ pitch and $80 \mu \mathrm{m}$ depth.

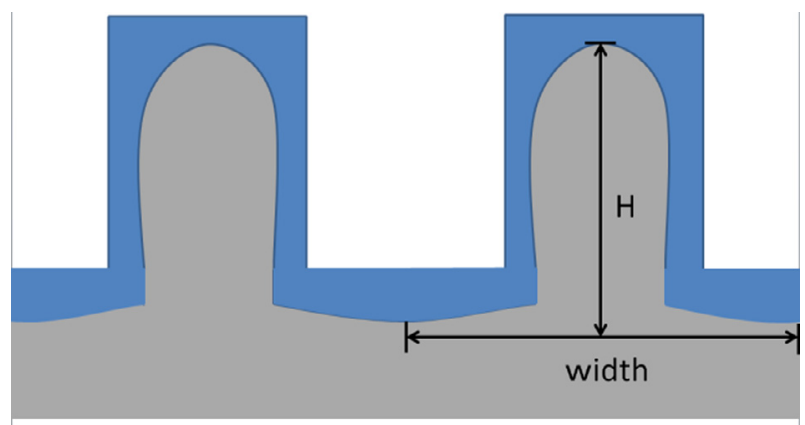

Figure 4. Schematic of imprinted sample's dimension.

\section{Results and discussion}

\subsection{Interface patterning}

To evaluate the deformation of the interface between the upper and lower layers, the height, $H$, was defined as shown in Figure 4. Four samples with different upper layer's thickness $(2.8,2.3,1.8$, and $1.5 \mu \mathrm{m})$ were prepared. Thickness of the lower layer was set to be $80 \mu \mathrm{m}$ for all samples. Figure 5 shows cross sectional images of the samples after the first imprinting. The interface height $H$ of each sample was 1.4, 2.7, 3.4, and $4.0 \mu \mathrm{m}$. As the thickness of the upper layer decreased, the interface height was increased.

Next, the samples were pressed by flat surface. The cross sectional views after pressing are shown in Figure 6. The interface height $H$ was $1.6,1.2,0.2$, and $1.7 \mu \mathrm{m}$ for each sample. The higher $H$ was found for samples with the thinnest and the thickest upper layers. The change of the interfacial pattern height after the first and the second imprinting was plotted in Figure 7. It is found that the pattern height after the first imprint showed a linear relationship, while the height after the second imprint resulted in a complicated manner. It is estimated that there are two modes of deformation in areas where initial thickness of the upper layer is smaller or larger than $1.7 \mu \mathrm{m}$. If the upper layer is enough thick, the interface pattern would not be disturbed during the second pressing, while if the upper layer is thin, buckling would occur near side wall portions, which could remain after the second pressing. Schematic of these behaviors are shown inside of the plot area.

In Figure 7, dashed curve was drawn which is estimated relationship between the initial thickness of the upper layer
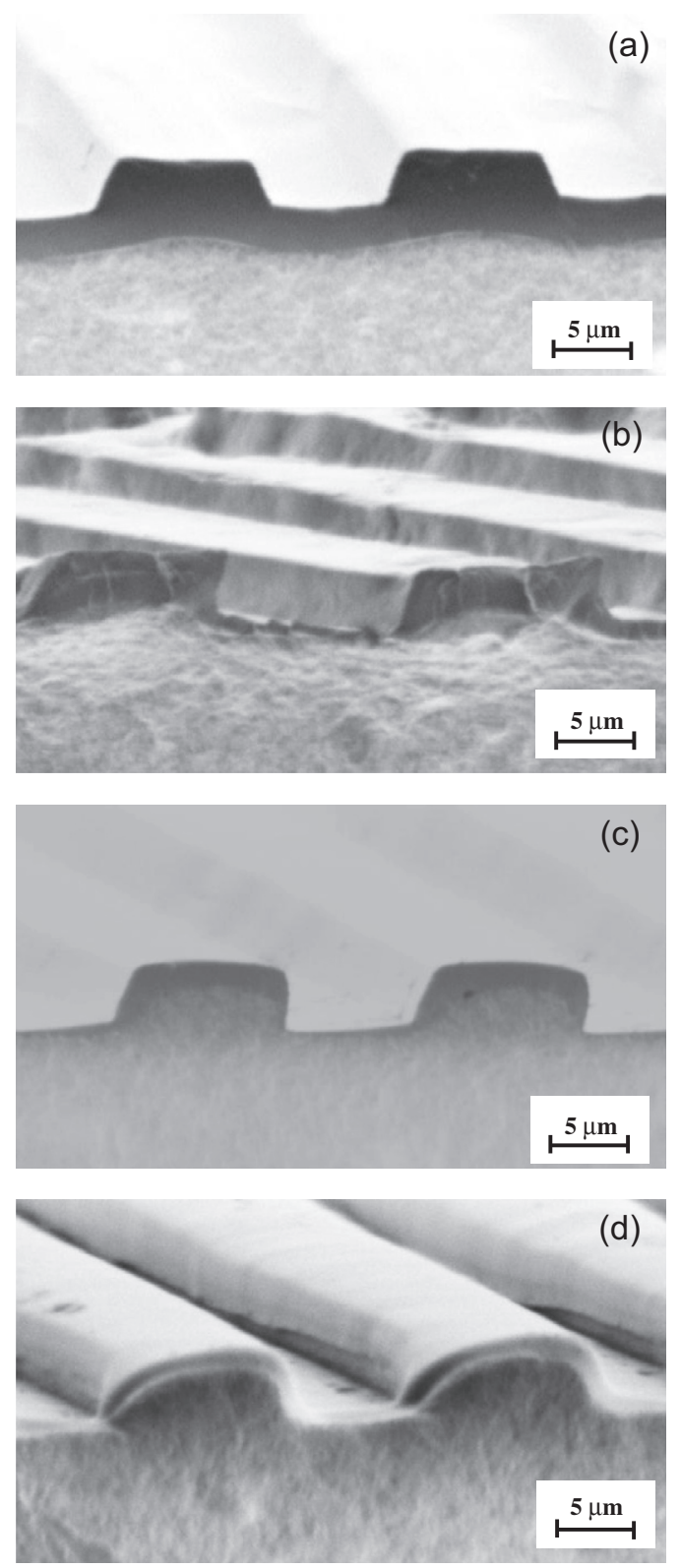

Figure 5. SEM images of interface patterns between the upper layer and lower layer with the pressure of $48 \mathrm{MPa}$. The thicknesses of upper layer are: (a) $2.8 \mu \mathrm{m}$, (b) $2.3 \mu \mathrm{m}$, (c) $1.8 \mu \mathrm{m}$, and (d) $1.5 \mu \mathrm{m}$. 

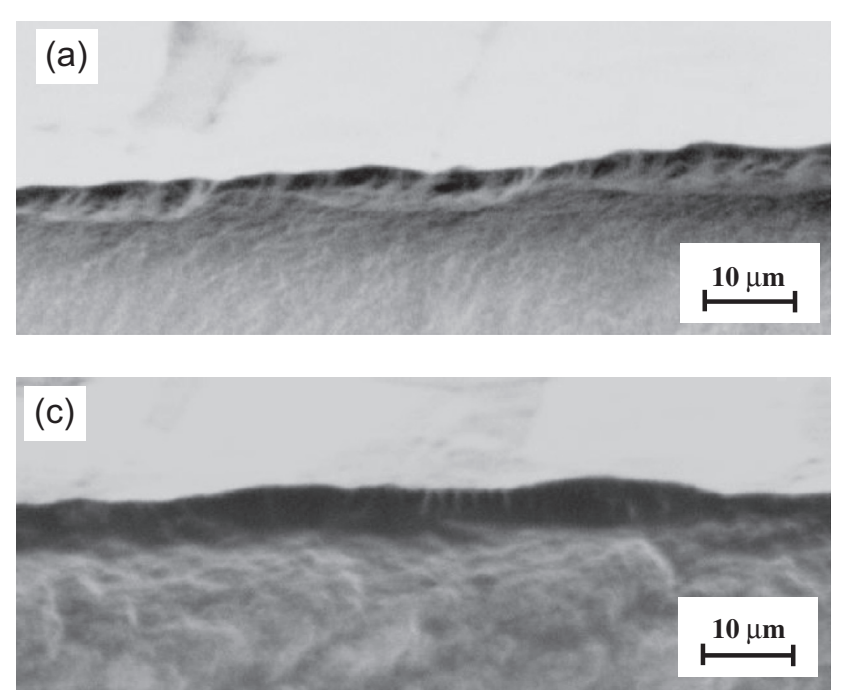

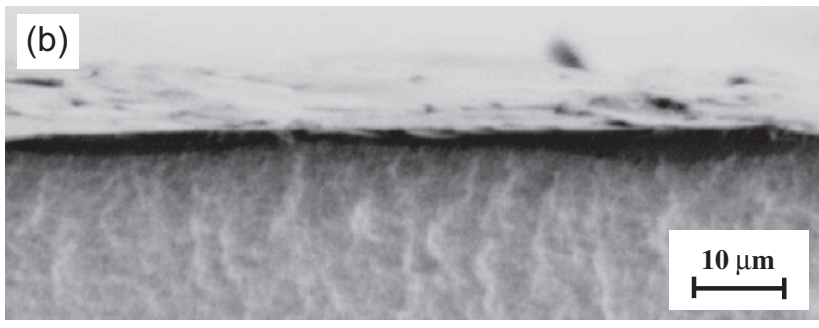

(d)

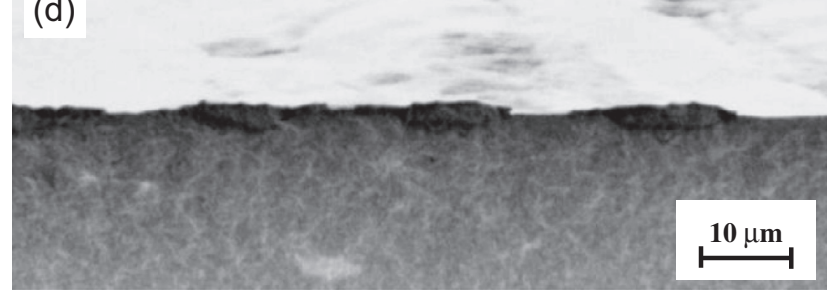

Figure 6. SEM images of interface patterns between the upper layer and lower layer after press with flat surface. The thicknesses of upper layer were (a) $2.8 \mu \mathrm{m}$, (b) $2.3 \mu \mathrm{m}$, (c) $1.8 \mu \mathrm{m}$, and (d) $1.5 \mu \mathrm{m}$.

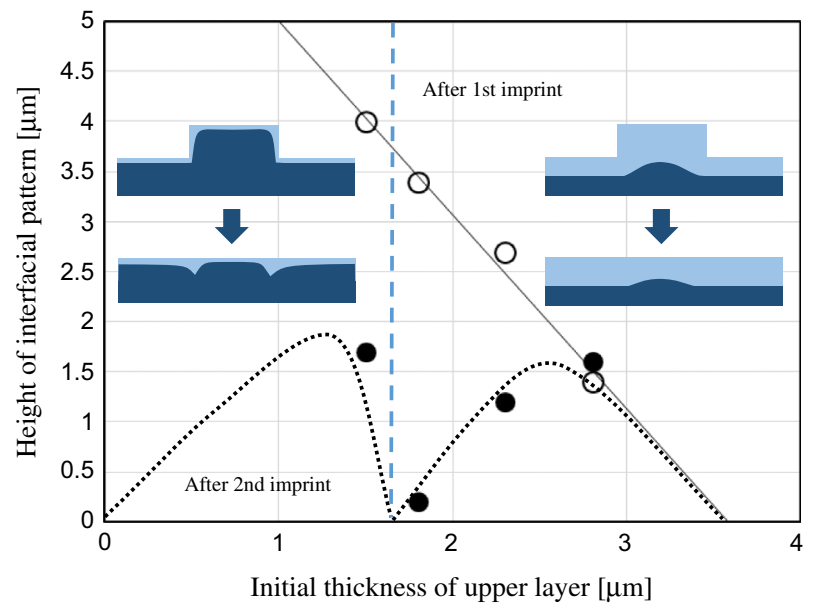

Figure 7. Relationships between initial thickness of upper layer and height of interfacial pattern. Empty circles show the pattern height after 1 st imprint, and solid circles show after 2 nd imprint. There are two modes of deformation, which are schematically drawn in the plot area.

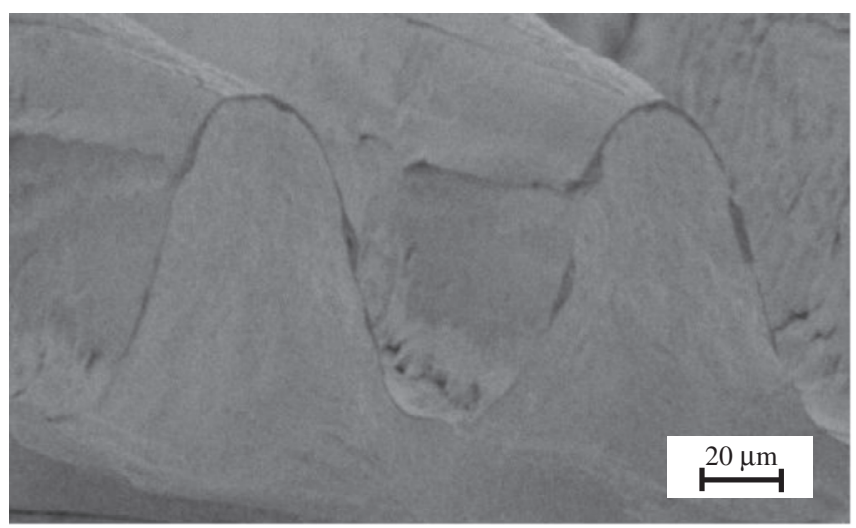

Figure 8. SEM image of interface pattern after the second imprinting with rougher mold. and the pattern height after the second imprint. We have only four points, and however, we can expect shape of the curve with two peaks. The curve should pass through the origin because the height should be zero if the initial thickness is zero. The curve also should be zero at the point where the initial thickness is about $3.5 \mu \mathrm{m}$ since the pattern height after the first imprinting was zero. We have shown the expected curve as described above. Of course, more data points should be collected to determine the exact relationship. It is also noted that the shape of this dashed curve is strongly depend on the mechanical properties of the upper and lower layers.

Next, polyimide mold with rougher pattern (Figure 3) was used instead of the flat surface for the second imprinting. In this experiment, upper layer of $1.5 \mu \mathrm{m}$ was chosen. The imprinting pressure was set to $60 \mathrm{MPa}$. Cross sectional SEM images are shown in Figure 8. The rougher patterns of the polyimide mold were clearly transcribed, and wavy pattern of $80 \mu \mathrm{m}$ height was obtained. The upper layer is shown as a black thin layer on the rough wavy surface. Between the black layer and grey layer below, which is $\mathrm{Al}_{2} \mathrm{O}_{3}$ compound layer, interface pattern can be observed. As shown in Figure 7, the interface looks wavy structure similar to the previous sample pressed by flat surface (Figure 6d). The interface height $H$ of the finer pattern left on the interface was $1.8 \mu \mathrm{m}$, which is close to the result of Figure $6 \mathrm{~d}, 1.7 \mu \mathrm{m}$. To obtain more interface height $H$ on the surface of hierarchical structures, or to obtain clearer interface, it should be needed to optimize the process conditions of the imprinting processes. Optimization of materials' deformation properties of both upper and lower layers could be also necessary to control the interfacial pattern, which governs contact angle with a water droplet. The contact angle is related with hydrophobicity. To obtain superhydrophobic surface state, a hierarchical pattern with both clear fine and rough patterns is needed. This optimization is our next work. 


\section{Conclusion}

In this work, the multi-step imprinting is proposed to fabricate a multi-scale pattern. We prepared four kinds of different thickness of upper layer; (a) $2.8 \mu \mathrm{m}$, (b) $2.3 \mu \mathrm{m}$, (c) $1.8 \mu \mathrm{m}$, and (d) $1.5 \mu \mathrm{m}$. After the first imprinting with $5 \mu \mathrm{m}$ depth mold with line \& space pattern, interface height $H$ was increased with decrease of the initial thickness of the upper layer. After the second stage pressing with flat surface, the Interface height $H$ became (a) $1.6 \mu \mathrm{m}$, (b) $1.2 \mu \mathrm{m}$, (c) $0.2 \mu \mathrm{m}$, and (d) $1.7 \mu \mathrm{m}$. After the second imprinting with rougher mold, imprinted pattern of the first imprinting was still remained as the interfacial pattern. In the present results, the fine pattern after the second imprinting was not very clear. For further improvement, optimization of the deformation properties of both upper and lower layer is necessary. It is also needed to optimize process parameters such as imprinting temperature, pressure and holding time.

\section{Implications and influences}

The proposed process will enable fabrication of hierarchical micro patterns on a sheet material with low cost. We showed that a ceramic powder material can be used for the process so that inorganic sheet materials can be obtained by the process. The patterned ceramic sheets could be available for industrial areas which require a controlling technique of surface wettability.

Acknowledgements. This work was partly supported by JSPS KAKENHI Grant Number 2560028. We are also grateful for financial support from the Amada Foundation.

\section{References}

1. D.H. Ko, J.R. Tumbleston, K.J. Henderson, L.E. Euliss, J.M. DeSimone, R. Lopez, E.T. Samulski, Biomimetic microlens array with antireflective "moth-eye" surface, Soft Matt. 7 (2011) 6404-6407.

2. H.J. Ensikat, P. Ditsche-Kuru, C. Neinhuis, W. Barthlott, Superhydrophobicity in perfection: the outstanding properties of the lotus leaf, Beilstein J. Nanotechnol. 2 (2011) $152-161$.

3. S.Y. Chou, P.R. Krauss, P.J. Renstrom, Imprint of sub-25 nm vias and trenches in polymers, Appl. Phys. Lett. 67 (1995) 3114-3116.

4. Y. Xu, F. Tsumori, H. Kang, H. Miura, Fabrication of micro patterned ceramic structure by imprinting process, J. Jpn. Soc. Powder Powder Metal. 58 (2011) 673-678.

5. Y. Xu, F. Tsumori, H.G. Kang, H. Miura, Study on fabrication of micrometer patterned ceramic sheet with micro powder imprinting $(\mu \mathrm{PI})$ method, Adv. Sci. Lett. 12 (2012) $170-173$.

6. F. Tsumori, S. Hashimoto, M. Takahashi, Y. Xu, H. Kang, T. Osada, H. Miura, Micro imprinting process for surface patterning of ceramic sheet, Proc. Powder Metallurgy World Congress (2013) P-T6-72.

7. Y. Xu, F. Tsumori, T. Osada, H. Miura, Improvement of solid oxide fuel cell by imprinted micro patterns on electrolyte, Micro Nano Lett. 8 (2013) 571-574.

8. Y. Xu, F. Tsumori, S. Hashimoto, M. Takahashi, H. Kang, T. Osada, H. Miura, Improvement of solid oxide fuel cell by imprinted patterns on electrolyte, Proc. IEEE- Nano/Micro Engineered and Molecular Systems (2013) 887-889.

9. F. Tsumori, Y. Xu, H. Kang, T. Osada, H. Miura, Simulation of deformation of layered sheet during micro powder imprinting process, Proc. Computational Plasticity XII (2013) 1267-1273.

10. F. Tsumori, Y. Tanaka, Y. Xu, T. Osada, H. Miura, Development of improved solid oxide fuel cell electrolyte sheet by micro imprinting for layered material, Jpn J. Appl. Phys. 53 (2014) 06JK02.

11. Y. Xu, F. Tsumori, T. Toyooka, H. Kotera, H. Miura, Effects of resist thickness and viscoelasticity on the cavity filling capability in bilayer thermal embossing, Jpn J. Appl. Phys. 50 (2011) 06GK11.

Cite this article as: Tsumori F, Shen L, Osada T \& Miura H: Hierarchical patterning by multi-step micro-imprinting with layered materials Manufacturing Rev. 2015, 2, 10. 